\title{
I see happy people: attention bias towards happy but not angry facial expressions in Williams syndrome
}

Article

Accepted Version

Author's version of accepted manuscript

Dodd, H. F. and Porter, M. A. (2010) I see happy people: attention bias towards happy but not angry facial expressions in Williams syndrome. Cognitive Neuropsychiatry, 15 (6). pp. 549-567. ISSN 1464-0619 doi:

https://doi.org/10.1080/13546801003737157 Available at https://centaur.reading.ac.uk/33959/

It is advisable to refer to the publisher's version if you intend to cite from the work. See Guidance on citing.

Published version at: http://www.tandfonline.com/10.1080/13546801003737157

To link to this article DOI: http://dx.doi.org/10.1080/13546801003737157

Publisher: Taylor \& Francis

Publisher statement: This is an Author's Accepted Manuscript of an article published in Cognitive Neuropsychiatry in 2011 [copyright Taylor \& Francis], available online at: http://www.tandfonline.com/10.1080/13546801003737157

All outputs in CentAUR are protected by Intellectual Property Rights law, including copyright law. Copyright and IPR is retained by the creators or other copyright holders. Terms and conditions for use of this material are defined in the End User Agreement. 


\section{CentAUR}

Central Archive at the University of Reading

Reading's research outputs online 
Dodd, H.F., \& Porter, M.A. (2010). I see happy people: attention bias towards happy but not angry facial expressions in Williams syndrome. Cognitive Neuropsychiatry, 15 (6), 549 - 567. doi: $10.1080 / 13546801003737157$

This is an Author's Accepted Manuscript of an article published in Cognitive Neuropsychiatry in 2011

[copyright Taylor \& Francis], available online at: http://www.tandfonline.com/10.1080/13546801003737157

Corresponding author:

Helen Dodd

Email: helen.dodd@mq.edu.au 


\section{Acknowledgements}

The authors would like to thank the schools, families and individuals who participated, Dr Alan Taylor for his statistical advice, Dr Anina Rich for her advice on the design and Alex Antell for her help with data collection. 
Abstract

Introduction: Observations of behaviour and research using eye-tracking technology have shown that individuals with Williams syndrome (WS) pay an unusual amount of attention to other people's faces. The present research examines whether this attention to faces is moderated by the valence of emotional expression.

Method: Sixteen participants with WS aged between 13 and 29 years (Mean=19 years 9 months) completed a dot-probe task in which pairs of faces displaying happy, angry and neutral expressions were presented. The performance of the WS group was compared to two groups of typically developing control participants, individually matched to the participants in the WS group on either chronological age or mental age. General mental age was assessed in the WS group using the Woodcock Johnson Test of Cognitive Ability Revised (WJ-COG-R; Woodcock \& Johnson, 1989; 1990).

Results: Compared to both control groups, the WS group exhibited a greater attention bias for happy faces. In contrast, no between-group differences in bias for angry faces were obtained.

Conclusions: The results are discussed in relation to recent neuroimaging findings and the hypersocial behaviour that is characteristic of the WS population. 
Williams syndrome (WS) is a rare neurodevelopmental disorder caused by a hemizygous microdeletion of approximately 25 genes on the long arm of chromosome 7 at 7q11.23 (Ewart et al., 1993, Fryssira, Palmer, Hallidie-Smith, Taylor, Donnai, \& Reardon, 1997; Osborne, Li, Pober, Chitayat, Bodurtha, Mandel, et al., 2001). WS is associated with a mild to moderate intellectual impairment and a unique social-behavioural phenotype; Individuals with WS display outgoing, hypersocial behaviour, treat everyone as if they were their friend, and exhibit unusually intense eye-contact (Doyle, Bellugi, Korenberg, \& Graham, 2004; Järvinen-Pasley et al., 2008; Jones et al., 2000; Mervis et al., 2003). Research has consistently shown that hypersocial behaviour is a pervasive characteristic of the WS behavioural phenotype. Studies using parent-report questionnaires have found that individuals with WS, even as young as 13 months, are rated by their parents as more sociable towards strangers than typically developing children and children with other developmental disorders (Doyle et al., 2004; Dykens \& Rosner, 1999; Jones et al., 2000; Sarimski, 1997). This fascinating social behaviour, and the cognitive and neurological processes that underpin it, has attracted significant research interest, particularly in recent years (Haas et al., 2009; Järvinen-Pasley et al., 2008; Jawaid, Schmolck, \& Schulz, 2008; Plesa-Skwerer et al., 2009; Porter, Coltheart, \& Langdon, 2007; Riby \& Hancock, 2009a).

One area of interest for recent research has been the role of attention to faces in WS social behaviour. Initial evidence of atypical attention patterns in WS came from observations that young children with WS spend an unusual amount of time looking at strangers' faces (Jones et al., 2000; Mervis et al., 2003). Recent research using eye-tracking technology has since supported these observations, finding that, when individuals with WS view social scenes, they tend to look at the actor's faces for prolonged periods (Riby \& Hancock, 2009b; Riby \& Hancock, 2008). Interestingly, these researchers have found no evidence that faces capture attention atypically in WS (Riby \& Hancock, 2009b). Instead, it seems likely that individuals with WS have difficulty disengaging attention from faces (Brown et al., 2003; Cornish, Scerif, \& Karmiloff-Smith, 2007; Riby \& Hancock, 2009b). This growing body of research provides important evidence that individuals with WS spend 
an unusual amount of time looking at faces. It is currently unclear, however, whether the emotional valence of the face affects this looking behaviour. As happy emotional expressions generally signal social engagement and angry facial expressions generally signal social threat, emotional expression may be important in moderating attention to faces in WS.

Distinctive responses to positively and negatively valanced facial expressions have been found in WS on approach judgement tasks, where participants are asked to rate the approachability of a series of unfamiliar faces. This procedure was initially used by Bellugi, Adolphs, Cassady, \& Chiles (1999) who found that participants with WS rated unfamiliar people as more approachable than typically developing controls. Two studies have subsequently extended these findings by asking participants to rate the approachability of faces displaying different expressions of emotion. Using angry, disgusted, fearful, happy, sad and neutral facial expressions, Frigerio et al. (2006) found that WS participants rated happy faces as more approachable than controls and all other faces as less approachable than controls. Porter et al. (2007) attempted to replicate this finding but found that, when emotion recognition was controlled for, WS participants made similar approach judgements to controls regardless of emotional expression. Importantly, however, both studies found that WS participants rated happy faces as significantly more approachable than angry faces, demonstrating that emotional expression can be an important determinant of social judgement in WS.

Further evidence that emotional valence is important in the processing of social stimuli in WS comes from neuroimaging research (Meyer-Lindenberg et al., 2005) and research assessing physiological arousal (Plesa-Skwerer et al., 2009). Meyer-lindenberg et al. (2005) examined amygdala activation whilst participants completed a matching task with images of angry or fearful faces and threatening scenes. Relative to typically developing controls, the WS group exhibited greater amygdala activation in response to the threatening scenes but attenuated amygdala activation in response to the negative faces. Consistent with this later finding, Plesa-Skwerer et al. (2009) assessed physiological arousal in a group of individuals with WS whilst they viewed a range of facial expressions and found that, relative to typically developing and intellectual impaired controls, 
the WS group were hypoaroused by angry expressions. In contrast, no significant group differences in arousal were found for happy expressions. These findings suggest that individuals with WS are not aroused by angry facial expressions and may not, therefore, process angry faces as threatening.

With a view to comparing neural responses to positively and negatively valanced social stimuli in WS, Haas et al. (2009) collected data using fMRI and ERP whilst participants completed a gender discrimination task with happy, fearful, neutral and scrambled faces. The results showed that, relative to typically developing controls, the WS group exhibited elevated amygdala reactivity to happy faces but not fearful faces. Furthermore, the ERP findings indicated an exaggerated response in the WS group when viewing happy faces and an attenuated response in the WS group when viewing fearful faces. These findings are consistent with those of Meyer-Lindenberg et al. (2005) and Plesa-Skwerer et al. (2009) in demonstrating attenuated amygdala activation in response to negative facial expressions. Importantly, however, these findings also provide the first evidence of elevated amygdala reactivity in WS in response to happy faces and the first evidence of a dissociation in neural activation for positive and negative facial expressions of emotion.

Taken together, the research discussed suggests that valence has an important influence on responses to social-emotional stimuli in WS. It seems likely, therefore, that attention to faces may be moderated by emotional expression. The present research explores this hypothesis by examining whether individuals with WS are biased to attend to happy facial expressions and angry facial expressions using a dot-probe task.

The dot-probe paradigm is commonly used to assess attention bias. This paradigm has been used extensively to demonstrate attention biases to threat in anxious populations (e.g. Bar-Haim, Lamy, Pergamin, Bakermans-Kranenburg, \& van ljzendoorn, 2007; Mogg, Garner, \& Bradley, 2007; Mogg, Philippot, \& Bradley, 2004; Roy et al., 2008). In contrast, there is little evidence from dotprobe tasks that typically developing adults and children are biased to attend to happy or angry emotional expressions over neutral expressions (Bar-Haim et al., 2007; Bradley, Mogg, Falla, \& Hamilton, 1998; Hadwin, Donnelly, Richards, French, \& Patel, 2009; Isaacowitz, Wadlinger, Goren, \& 
Wilson, 2006; Peltola, Leppanen, Vogel-Farley, Hietanen, \& Nelson, 2009; Roy et al., 2008). In the dot-probe task a neutral stimulus and an emotional stimulus are presented simultaneously, followed immediately by a probe in the same location as either the emotional or neutral stimulus. Participants are instructed to respond to the probe as quickly as possible. This paradigm has been used to assess both within-subjects and between-subjects attention biases. A within-subjects bias is found when a group responds significantly faster to the probe when it follows an emotional stimulus (congruent trial) than a neutral stimulus (incongruent trial). A between-subject bias occurs when significant differences in the size of the bias (congruent trials - incongruent trials) are found between two or more groups.

Although the dot-probe task has been used extensively, there is some debate regarding which components of attention the task measures. Derryberry and Reed (2002) highlighted that a faster response time on congruent than incongruent trials could occur because the threat image captures attention which leads to a faster response time on congruent trials and/or because it is difficult to disengage attention from the threat image which leads to a slower response time on incongruent trials. Koster, Crombez, Verschuere, \& De Houwer (2004) explored these alternatives in a study with typically developing adults by including a baseline condition in which both images were neutral. By comparing congruent and incongruent trials to the neutral condition it was possible to differentiate between vigilance and disengage effects. Koster et al. (2004) found evidence for disengage effects and no evidence for enhanced vigilance for threat. Other research that has investigated vigilance and disengage effects has supported these findings, reporting only disengage effects (Salemink, van den Hout, \& Kindt, 2007; Yiend \& Mathews, 2001). As this is the first study to examine attention to emotional faces in WS, either vigilance or disengage effects are possible. However, there is some evidence from eye-tracking research that individuals with WS may have difficulty disengaging attention from faces in general (Riby \& Hancock, 2009b) and also evidence that individuals with WS may have difficulty with the shifting component of attention (Brown et al., 2003; Cornish et al., 2007). 


\section{Aims and hypotheses}

The primary aim of this research was to examine attention bias for happy and angry facial expressions in WS and two groups of typically developing controls, matched to the WS group on mental age or chronological age. Within-subjects effects were examined by comparing congruent and incongruent trials on the dot-probe task for both happy and angry facial expressions. Betweensubjects effects were examined by comparing overall bias for happy and angry faces in the WS group to overall bias in the control groups.

Based on the research outlined above, it was hypothesised that the WS group would exhibit a within-subjects attention bias towards happy faces but not angry faces. No within-subjects attention bias for either happy or angry faces was anticipated in the control groups. In keeping with these hypotheses, a significant between-subjects bias was expected for happy faces but not angry faces, with the WS group exhibiting a larger bias towards happy faces than both control groups. Following Koster et al. (2004) a neutral condition, in which two neutral faces are presented, was also included such that vigilance and disengage effects could be distinguished. Further, to control for group differences in emotion recognition ability, an emotion recognition task was conducted after the dot-probe task had been completed.

Method

The study involved 48 participants: 16 participants with WS and 32 typically developing participants, divided into mental age matched and chronological age matched control groups. Demographic data for each group is shown in Table 1.

Williams syndrome group.

Participants were sixteen individuals with WS ( $N=16,9$ male) aged between 13 years 7 months and 29 years 1 month with a mean age of 19 years 9 months. All participants had received a diagnosis of WS following a positive FISH test showing deletion of the elastin gene at 7q11.23 (Fryssira et al., 1997) and exhibited the typical WS phenotype (Bellugi, Lichtenberger, Jones, Lai, \& St, 2000; Dykens, 2003). Participants were recruited through the Australian Williams Syndrome 
Association. Due to the attentional demands of the task, only individuals with a mild to moderate intellectual impairment who had a mental age of at least 6 years as assessed using the WoodcockJohnson Test of Cognitive Ability - Revised (WJ-COG-R; Woodcock \& Johnson, 1989, 1990) were invited to participate. The mental age of the participants with WS ranged from 6.2 years to 10.58 years, with a mean of 8.05 . IQ scores ranged from 53 to 77 representing the mild to moderate impairment range that is typical of individuals with WS.

\section{Mental age comparison group.}

Sixteen typically developing children whose chronological age and sex matched the mental age and sex of individuals in the WS group, were recruited through primary schools in the Sydney area. The mean age of the mental-age matched control groups was 8 years 2 months. All control participants were considered to be 'typically developing' by their parents and teachers. Children with a developmental disorder, clinical diagnosis, or any history of atypical development were not selected to participate. Using chronological age as a proxy for mental age, the mental age comparison group were closely matched to the WS group, $\mathrm{t}(15)=-0.527, p=0.606$.

\section{Chronological age comparison group.}

Sixteen typically developing participants, individually matched to the WS participants on chronological age and sex, were recruited via a university-administered register of teenagers and young adults who are interested in participating in research. The mean age of the chronological age match control group was 19 years 9 months. Again, all participants were considered to be 'typically developing'; participants with a history of developmental disorder, clinical diagnoses or any history of atypical development were not selected to participate. The chronological age comparison group were closely matched to the WS group on chronological age, $\mathrm{t}(15)=-1.721, p=1.06$.

$$
\text { [Insert Table } 1 \text { here] }
$$

\section{Design}

The dot-probe task was based on that used in previous studies (e.g. Waters, Lipp, \& Spence, 2004) and included a total of 288 experimental trials divided into twelve blocks of twenty-four trials. 
Each block incorporated sixteen critical trials: eight in which an angry face was presented with a neutral face and eight in which a happy face was presented with a neutral face. Emotion (happy/angry), emotion position (left/right) and probe position (left/right) were manipulated such that each block included four angry-congruent trials, four angry-incongruent trials, four happycongruent trials and four happy-incongruent trials. A congruent trial was defined as a trial in which the probe was located in the same position as the emotional stimulus. Conversely, an incongruent trial was a trial in which the probe was located in the same position as the neutral stimulus. The position of the emotion and probe were counterbalanced within conditions. In addition to the sixteen critical trials, each block also included eight neutral trials, in which two neutral images were presented, to provide a baseline for participants' reaction time when no emotion was present. On these trials the position of the probe was also counterbalanced.

During each trial a pair of images of the same actor was presented. The experiment was designed such that a face pair for each actor was seen once in each block and in each possible condition an equal number of times throughout the experiment. Trials were randomized within blocks for each participant.

\section{Apparatus and materials}

Images from twenty-four different actors (12 male, 12 female) were taken from the Karolinska Directed Emotional faces (KDEF; Lundqvist, Flykt, \& Ohman, 1998), a set of 4900 pictures of actors displaying facial expressions, designed for use in psychological research. Three images were selected for each actor: a neutral expression; a happy expression; and an angry expression. These images were then used to create the image pairs required for each condition.

The original stimuli were adjusted to grayscale and a grey border was added to cover the background and hair. The images were adjusted to ensure that the mean and standard deviation of luminosity for the images in each image pair were closely matched.

The dot-probe task was programmed using DMDX (Forster \& Forster, 2003) and presented on a 15" MacBook Pro operating Windows XP SP3. 


\section{Procedure}

Informed consent was obtained from the participants or their parents, as appropriate. The study was approved by the Macquarie University Human Ethics Committee. Participants were tested individually in a quiet room either in their home or at the University. In total, the experimental measures took approximately 25 minutes to complete, breaks were provided as necessary. Participants sat approximately $60 \mathrm{~cm}$ from the computer screen.

In order to keep the attention task as simple as possible, a probe-detection task was chosen over a probe classification task. The dot-probe procedure was based on that used in previous research with children aged seven years and above (Mogg, Philippot et al., 2004; Roy et al., 2008; Waters, Mogg, Bradley, \& Pine, 2008). Each trial began with a black fixation cross in the centre of a white background for 500 ms followed by presentation of the two images on the left and right side of the fixation cross for $500 \mathrm{~ms}$. The inner edge of each image was $1.6 \mathrm{~cm}$ away from the fixation cross and the images measured $8 \mathrm{~cm} \times 6 \mathrm{~cm}$. The centre points of the two images were separated horizontally by a visual angle of approximately $9^{\circ}$. The two images were then followed immediately by a probe presented in the centre of the space occupied by one of the two previous images. The probe was a light grey dot measuring $0.4 \mathrm{~cm}$ diameter. The probe was presented $4.4 \mathrm{~cm}$ away from the fixation cross. Participants were told to press the shift key that corresponded to the side the probe was on as quickly as possible. The probe remained on the screen until a response had been made or until 10 seconds had passed. The participants' response to the probe, or the timeout of the probe, was followed by a 100 tick (approximately $1672 \mathrm{~ms}$ ) inter-trial interval. The fixation cross remained on the screen throughout each 90 second block. The experiment ran continuously within blocks then, at the end of each block, participants were told that they could take a break. They were instructed to press the spacebar when they were ready for the next block. At the start of the experiment, participants completed six practice trials and were given an opportunity to ask questions before the experimental trials began. Accuracy and reaction time (RT) data were recorded for all trials. 


\section{Emotion Recognition Task}

To ensure that participants were able to correctly identify the emotions expressed in the dot-probe task images, participants also completed a test of emotion recognition. To control for the possibility that completion of the emotion recognition task might affect attention allocation on the dot-probe task, the emotion recognition task was always completed after the dot-probe task. In the emotion recognition task, images of emotional faces were presented for $500 \mathrm{~ms}$ and participants were then asked to select from a list of written options (happy, sad, angry, scared, or neutral) how they thought the person in the image was feeling. Participants' ability to read each emotion label was checked at the start of the emotion recognition task. To minimize time constraints, thirty of the emotional images used in the dot-probe task (10 happy, 10 angry, 10 neutral) were randomly selected and presented to participants with an additional twenty images (10 sad and 10 scared). The order of images was randomized for each participant. Participants received a score out of ten for each of the three emotion categories used in the dot-probe task (happy, angry and neutral).

\section{Results}

\section{Data preparation - dot-probe task}

Similar to previous studies (Koster et al., 2004; Mogg, Bradley, Miles, \& Dixon, 2004), incorrect trials and trials with timing errors (defined as trials with RTs of $<200 \mathrm{~ms}$ or $>3000 \mathrm{~ms}$ ) were removed and a mean and standard deviation were calculated for each participant. RTs that were more than 2 standard deviations above each participant's mean were then also removed. The mean percentage of trials for which RTs were removed was $4.86 \%$ for the WS group, $4.25 \%$ for the CA group and 7.55\% for the MA group. The WS group did not differ from the CA group in the amount of RT data removed, $\mathrm{t}(15)=1.172, p=0.260$. Significant differences were found between the WS and MA group due to more incorrect responses in the MA group, $\mathrm{t}(15)=-2.548, p=0.022$. All further analyses were conducted with mean RT data.

Dot-probe task 
Table 2 shows the mean and standard deviation of RTs for each group (WS, CA, MA) on neutral, angry-congruent, angry-incongruent, happy-congruent and happy-incongruent trials ${ }^{1}$. A congruent trial was one in which the probe was located in the same position as the emotional image, an incongruent trial was one in which the probe was located in the same position as the neutral image.

For the following analyses the Bonferroni correction for multiple comparisons is applied where appropriate. A $p$-value that is statistically significant at $p<0.05$ but not at the corrected $p$ value is described as marginally significant. Cohen's $d$ effect size estimates are reported for each pairwise comparison.

\section{[Insert Table 2 here]}

A repeated-measures ANOVA was conducted with congruency (congruent/incongruent) and emotion (angry/happy) as within-subjects variables and group (WS, CA, MA) as a between-subjects variable. The results indicated a significant main effect of group, $F(2,45)=21.271, \mathrm{MSE}=$ 757193.22, $p<0.001$, but no significant main effect of congruency, $\mathrm{F}(1,45)=0.043, \mathrm{MSE}=18.408, p$ $=0.836$, or emotion, $F(1,45)=0.252, \mathrm{MSE}=34.062, p=0.618$. None of the two-way interactions were significant ( $p>0.05)$, but the three-way interaction between emotion, congruency and group was significant, $F(2,45)=4.514, \mathrm{MSE}=1090.211, p=0.016$. To explore this interaction, follow-up analyses were conducted for each emotion independently and then between emotions.

\section{Angry Bias: Congruent and Incongruent trials.}

Mean bias scores (incongruent trials - congruent trials) for angry faces are shown in Table 2. A repeated-measures ANOVA was conducted with congruency (congruent/incongruent) as a within subjects variable and group (WS, CA, MA) as a between-subjects variable. The results indicated a significant main effect of group, $F(2,45)=21.079, \mathrm{MSE}=376557.291, p<0.001$, but no significant main effect of congruency, $F(1,45)=1.003, \mathrm{MSE}=255.617, p=0.322$, and no significant group by congruency interaction, $\mathrm{F}(2,45)=0.160, \mathrm{MSE}=40.753, p=0.853$. No evidence of within-subjects or between-subjects biases was therefore present for angry faces. 
Happy Bias: Congruent and Incongruent trials.

Mean bias scores (incongruent trials - congruent trials) for happy faces are shown in Table 2. A repeated-measures ANOVA was conducted with congruency (congruent/incongruent) as a withinsubjects variable and group (WS, CA, MA) as a between-subjects variable. The results indicated no significant main effect of congruency, $\mathrm{F}(1,45)=0.240, \mathrm{MSE}=98.415, p=0.627$, but a significant main effect of group, $F(2,45)=21.303, \mathrm{MSE}=380641.439, p<0.001$, and a significant group by congruency interaction, $\mathrm{F}(2,45)=5.743, \mathrm{MSE}=2356.414, p=0.006$. To explore this interaction, separate analyses were conducted within groups and between groups.

T-tests were conducted to examine the effect of congruency for each group independently. A bonferroni corrected $p$-value of $0.017(0.05 / 3)$ was used to indicate statistical significance. The WS group were faster on congruent than incongruent trials but this did not reach significance, $t(15)=-$ 2.001, $p=0.064(d=0.25)$. The MA group were faster on incongruent than congruent trials but this did not reach significance, $t(15)=2.040, p=0.059(d=0.08)$. No significant effect of congruency was found for the CA group, $\mathrm{t}(15)=1.597, p=0.131(d=0.08)$.

To examine whether the bias differed significantly between groups, a bias score was calculated for each participant by subtracting their mean RT on congruent trials from their mean RT on incongruent trials (see Table 2). A positive number therefore indicated a bias towards happy faces and a negative number a bias away from happy faces. T-tests were then conducted to compare the WS group to both control groups on overall bias, a p-value of $0.025(0.05 / 2)$ was used to indicate statistical significance. The bias exhibited by the WS group differed significantly from that found in the CA group, $\mathrm{t}(30)=2.398, p=0.023(d=0.85)$ and the MA group, $\mathrm{t}(30)=2.661, p=0.012(d=0.94)$. Happy Bias: Engage vs Disengage effects.

The findings suggest an attention bias to happy faces was present in the WS group. Consequently, following Koster et al. (2004) further t-tests were conducted to examine whether this bias was due to attention engagement or disengagement by comparing the neutral condition with the congruent and incongruent condition. Engage effects occur when a significant difference is 
found between neutral trials and congruent trials, suggesting that the emotional image is capturing the attention of the participants. In contrast, disengage effects occur when a significant difference is found between reaction time on neutral trials and reaction time on incongruent trials, suggesting that participants are having difficulty disengaging their attention from the emotional image to respond to a probe in a different location. A p-value of $0.025(0.05 / 2)$ was used to indicate statistical significance. A marginally significant difference was found for the WS group between incongruent trials and neutral trials, $\mathrm{t}(15)=-2.171, p=0.046(d=0.19)$, but no difference was found between neutral and congruent trials, $\mathrm{t}(15)=0.963, p=0.598(d=0.06)$. To examine whether there was any evidence of engage or disengage effects in the typically developing control groups, these analyses were also conducted for the MA group and CA group independently. None of the effects reached significance, $p>0.2$.

\section{Bias, age and gender}

To explore whether overall bias for happy or angry faces was related to either chronological age or mental age, Pearson product moment correlation coefficients were calculated. For the sample as a whole and each group in isolation, no significant correlations between happy or angry bias scores and either mental age or chronological age were found $(p>0.1)$. Further, independent samples t-tests were conducted to examine the effect of gender on happy and angry bias. No significant affect of gender of found for the entire sample or any group in isolation $(p>0.1)$.

\section{Emotion Recognition Task}

The mean number of images correctly labeled for each emotion and group are shown in Figure 1. A repeated-measures ANOVA was conducted with emotion (happy/angry/neutral) as a within-subjects variable and group (WS, CA, MA) as a between-subjects variable. The results indicated a significant main effect of emotion, $F(2,90)=39.712$. MSE $=61.523, p<0.001$, but no significant main effect of group, $F(2,45)=2.167, \mathrm{MSE}=7.174, p=0.126$, and no significant group by emotion interaction, $\mathrm{F}(4,90)=1.558, \mathrm{MSE}=2.153, p=0.199$. Follow-up $\mathrm{t}$-tests indicated that all participants were significantly less accurate at recognizing anger than both happy, $t(47)=-7.881$, 
$p<0.001$, and neutral, $\mathrm{t}(47)=-5.283, p<0.001$. and significantly less accurate at recognizing neutral than happy, $\mathrm{t}(47)=-3.567, p=0.001$.

\author{
[Insert Figure 1 here]
}

Discussion

There is consistent evidence that individuals with WS spend an unusual amount of time looking at faces (Jones et al., 2000; Mervis et al., 2003, Riby \& Hancock, 2008, 2009a). However, to date, there has been no consideration of how the valence of emotional expression might affect this attention to faces. Consequently, the present research examined whether individuals with WS are biased to attend to happy and angry facial expressions using a dot-probe task. It was hypothesised that the WS group would show a within-subjects attention bias towards happy faces and that this bias would differ significantly from the pattern of responding found in typically developing controls matched on mental or chronological age (between-subjects bias). In contrast, it was hypothesised that the WS group would show no within-subjects or between-subjects bias for angry faces. In general, the results provided support for these hypotheses.

\title{
Happy faces
}

The overall pattern of results was consistent with the hypotheses. Some evidence of a within-subjects attention bias to happy faces was found in the WS group, but not in either control group. Furthermore, a significant between-subjects bias was found between the WS group and both control groups. Although the pattern of results for the within-subjects bias was in keeping with the hypotheses, the bias for the WS group did not reach significance and the effect size estimate was small, suggesting that a larger sample would be required for this within-subjects bias to reach statistical significance. In contrast, the evidence for a between-subjects bias was very clear; the WS group exhibited a bias for happy faces that was significantly different to that found in both control groups. These later comparisons were also supported by large effect size estimates.

By including a neutral condition it was possible to examine whether the bias to happy faces in the WS group was likely due to attentional capture or difficulty disengaging attention (Koster et 
al., 2004). The results suggest that the bias was likely driven by difficulties disengaging attention, rather than attentional capture. This is consistent with research with the typically developing population suggesting that attention biases are typically driven by disengage effects (Koster et al., 2004; Salemink et al., 2007; Yiend \& Mathews, 2001) and also with evidence that individuals with WS have difficulty disengaging their attention in general (Brown et al., 2003; Cornish et al., 2007) and from faces in particular (Riby \& Hancock, 2009b).

Angry faces

The hypotheses in relation to angry faces were supported; there was no evidence of a within-subjects attention bias in any of the groups and no evidence of a between-subjects bias between the groups.

\section{Emotion recognition}

As the images were presented briefly during the dot-probe task, an emotion recognition task was conducted to ensure that participants were able to extract accurate emotional information from the images in 500ms. No significant group differences in emotion recognition were found. This finding is important as it suggests that group differences in emotion recognition were not responsible for the pattern of results on the dot-probe task. The result is, however, inconsistent with previous research reporting that individuals with WS are impaired at emotion recognition relative to their chronological age matched peers (Plesa-Skwerer, Faja, Schofield, Verbalis, \& Tager-Flusberg, 2006; Porter et al., 2007). This inconsistency may be related to differences in the methodology of the emotion recognition tasks and the emotional stimuli used. For example, in the present research, black and white images were presented for $500 \mathrm{~ms}$ and participants had to label the emotion after the image had left the screen. In contrast, in previous research, participants have viewed colour photographs for as long as they needed to and labelled the emotion with the image present. Furthermore, in the present research, all groups performed reasonably well on the emotion recognition task. It seems possible, therefore, that it is easier to recognise the emotions portrayed in the KDEF images than the images used in previous research. 
In summary, the results suggest that individuals with WS are significantly more likely to attend to happy faces, but not angry faces, than typically developing controls and that this effect is not caused by group differences in emotion recognition ability. These findings suggest that individuals with WS not only pay a lot of attention to faces in general (Jones et al., 2000; Mervis et al., 2003; Riby \& Hancock, 2009a) but may be biased to attend to happy faces in particular. Attention bias, the amygdala and social behaviour in WS

As discussed, Haas et al. (2009) recently presented evidence that individuals with WS exhibit elevated amygdala activation in response to happy but not angry faces. These researchers discuss that this elevated amygdala activation found in WS in response to happy faces may serve to increase attention to happy faces. In finding evidence of an attention bias for happy faces, but not angry faces, in WS, the present findings provide some support for this hypothesis. However, in order to directly link amygdala activation with attention bias in WS, it will be necessary for future research to use $\mathrm{fMRI}$ in combination with behavioural measures of attention bias.

In general, there is little evidence that typically developing adults and children are biased to attend to happy or angry emotional expressions over neutral expressions (Bar-Haim et al., 2007; Bradley, Mogg, Falla, \& Hamilton, 1998; Hadwin, Donnelly, Richards, French, \& Patel, 2009; Isaacowitz, Wadlinger, Goren, \& Wilson, 2006; Mogg, Millar, \& Bradley, 2000; Mogg, Philippot et al., 2004; Peltola, Leppanen, Vogel-Farley, Hietanen, \& Nelson, 2009; Roy et al., 2008). It seems plausible, therefore, that the attention bias for happy faces found in the WS group may be related to the hypersocial behaviour observed in this population. Furthermore, as recent research has demonstrated that subtle attention biases for threatening stimuli may play an important role in the onset of anxiety symptoms (MacLeod, Rutherford, Campbell, Ebsworthy, \& Holker, 2002; See, MacLeod, \& Bridle, 2009), it seems possible that a subtle attention bias for happy faces could be related to the development of hypersocial behaviour. One difficultly with this hypothesis is that, in the present research, although a significant bias was found in WS at the group level, a subgroup of participants with WS did not exhibit any bias for happy faces. Yet all of the WS participants displayed 
the social personality that is typical of this population. There was little evidence that this heterogeneity within the WS group was related to chronological age, mental age or gender, although care must be taken in interpreting these correlations due to the small sample size. An important goal for future research will, therefore, be to explore individual differences in attention bias in relation to measures of social behaviour, genetic variation, cognitive abilities and neurological factors such as amygdala activation (c.f. Haas et al., 2009) and amygdala volume (c.f. Martens, Wilson, Dudgeon, \& Reutens, 2009).

\section{Attention bias and anxiety in WS}

The dot-probe paradigm is often used to examine attention bias in typically developing children and adults who are at risk of developing anxiety disorders or diagnosed with clinical anxiety disorders. There is now extensive evidence that attention biases for threatening stimuli are found in these populations (Bar-Haim et al., 2007).

This research is of relevance here because, in addition to atypical social behaviour, the WS behavioural phenotype is associated with increased risk for certain anxiety disorders. For example, Leyfer, Woodruff-Borden, Klein-Tasman, Fricke and Mervis (2006) assessed a large sample of children with WS and found unusually high rates of Generalised Anxiety Disorder and Specific Phobia, relative to the typically developing population. Interestingly, there is currently no evidence that rates of Social Phobia are elevated in WS (Dodd \& Porter, 2009; Leyfer et al., 2006). Given the link between attention bias for threatening stimuli and anxiety risk in the typically developing population, recent research has examined whether WS is associated with an attention bias for threatening scenes (Dodd \& Porter, submitted). Using a dot-probe task closely comparable to that used in the present research, clear evidence for an attention bias for threatening scenes was found in WS. As no evidence of an attention bias for angry faces was found in the present research, it appears that this threat-related bias does not extend to threatening faces. This dissociation in bias for threatening scenes and threatening faces is highly consistent with both the pattern of amygdala activation reported by Meyer-Lindenberg et al. (2005) and the profile of anxiety risk reported in WS. 


\section{Methodological considerations and directions for future research}

The present findings need to be considered within the context of a number of methodological considerations. With regards the dot-probe task, the images were displayed for $500 \mathrm{~ms}$, which is consistent with a number of previous studies (Mogg, Philippot et al., 2004; Roy et al., 2008; Waters et al., 2008) and eye-tracking evidence suggesting that behavioural responses recorded after 500ms are representative of initial allocation of attention (Mogg et al., 2000). It remains possible, however, that different results may be found at different presentation times, for example, if the stimuli were presented subliminally. Additionally, in the present task angry rather than fearful faces were used. Angry faces were chosen as anger may represent a more direct threat than fear (Mogg et al., 2000). It would be of interest, however, to replicate these findings using fearful faces.

Due to the cognitive demands of the task, we decided to only invite individuals with WS who have, at worst, a mild to moderate intellectual impairment and a mental age of 6 years to participate. As WS is a rare disorder, this resulted in a small sample size. Participants were recruited nationally to ensure the sample size was as large as possible and the resulting participant numbers are comparable to many recent studies conducted with this population (Krajcsi, Lukacs, Igacs, Racsmany, \& Pleh, 2009; Riby \& Hancock, 2009a; Vicari, Bellucci, \& Carlesimo, 2006). It would, however, be useful for future research to replicate these findings in other samples of individuals with WS. Finally, it was not possible in this circumstance to use eye-tracking as well as behavioural measures of spatial attention, however, as eye-tracking technology allows for real-time tracking of eye-movements, use of this procedure in the future may provide further insight into patterns of attention engagement and disengagement in WS in response to social-emotional stimuli.

\section{Conclusion}

The present research provides the first evidence that individuals with WS may be biased to attend to happy faces. No evidence for an analogous attention bias for angry faces was found. Important areas of interest for future research include explicitly examining the relationship between amygdala 
activation, attention bias and social behavior in WS and also extending the present findings using other emotional expressions such as fear and combining behavioral measure of attention bias with eye-tracking methodologies.References

Bar-Haim, Y., Lamy, D., Pergamin, L., Bakermans-Kranenburg, M. J., \& van ljzendoorn, M. H. (2007). Threat-related attentional bias in anxious and nonanxious individuals: A meta-analytic study. Psychological Bulletin, 133, 1-24.

Bellugi, U., Adolphs, R., Cassady, C., \& Chiles, M. (1999). Towards the neural basis for hypersociability in a genetic syndrome. Neuroreport, 10, 1653-1657.

Bellugi, U., Lichtenberger, L., Jones, W., Lai, Z., \& St (2000). I. The neurocognitive profile of Williams syndrome: A complex pattern of strengths and weaknesses. Journal of Cognitive Neuroscience, 12, 7-29.

Bradley, B. P., Mogg, K., Falla, S. J., \& Hamilton, L. R. (1998). Attentional bias for threatening facial expressions in anxiety: Manipulation of stimulus duration. Cognition \& Emotion, 12, 737753.

Brown, V., Johnson, M. H., Paterson, S. J., Gilmore, R., Longhi, E., \& Karmiloff-Smith, A. (2003). Spatial representation and attention in toddlers with Williams syndrome and Down syndrome. . Neuropsychologia, 41, 1037-1046.

Cornish, K., Scerif, G., \& Karmiloff-Smith, A. (2007). Tracing syndrome-specific trajectories of attention across the lifespan. Cortex, 43, 672-685.

Derryberry, D., \& Reed, M. A. (2002). Anxiety-related attentional biases and their regulation by attentional control. Journal of Abnormal Psychology, 111, 225-236.

Dodd, H. F., \& Porter, M. A. (2009). Psychopathology in Williams syndrome: The effect of individual differences across the life span. Journal of Mental Health Research in Intellectual Disabilities, 2, 89-109.

Dodd, H. F., \& Porter, M. A. (submitted). Attention bias to threat in williams syndrome. 
Doyle, T. F., Bellugi, U., Korenberg, J., \& Graham, J. (2004). "Everybody in the world is my friend" Hyperscociability in young children with Williams syndrome. American Journal of Medical Genetics, 124A, 263-273.

Dykens, E. M. (2003). The Williams syndrome behavioral phenotype: The 'whole person' is missing. Current Opinion in Psychiatry, 16, 523-528.

Dykens, E. M., \& Rosner, B. A. (1999). Refining behavioral phenotypes: Personality-motivation in Williams and Prader-willi syndromes. American Journal on Mental Retardation, 104, 158169.

Ewart, A., Morris, C. A., Atkinson, D., Jin, W., Sternes, K., Spallonem, P., et al. (1993). Hemizygosity at the elastin locus in a developmental disorder, Williams syndrome. Nature Genetics, 5, 11-16.

Forster, K. I., \& Forster, J. C. (2003). DMDX: A windows display program with millisecond accuracy. Behavior Research Methods, Instruments \& Computers, 35, 116-124.

Frigerio, E., Burt, D., Gagliardi, C., Cioffi, G., Martelli, S., Perrett, D. I., et al. (2006). Is everybody always my friend? Perception of approachability in Williams syndrome. Neuropsychologia 44, 254-259.

Fryssira, H., Palmer, R., Hallidie-Smith, K. A., Taylor, J., Donnai, D., \& Reardon, W. (1997). Fluorescent in situ hybridisation (FISH) for hemizygous deletion at the elastin locus in patients with isolated Supravalvar Aortic Stenosis. Journal of Medical Genetics, 34, 306-308.

Haas, B. W., Mills, D., Yam, A., Hoeft, F., Bellugi, U., \& Reiss, A. (2009). Genetic influences on sociability: Heightened amygdala reactivity and event-related responses to positive social stimuli in Williams syndrome. Journal of Neuroscience, 29, 1132-1139.

Hadwin, J. A., Donnelly, N., Richards, A., French, C. C., \& Patel, U. (2009). Childhood anxiety and attention to emotion faces in a modified stroop task. British Journal of Developmental Psychology, 27, 487-494. 
Isaacowitz, D. M., Wadlinger, H. A., Goren, D., \& Wilson, H. R. (2006). Is there an age-related positivity effect in visual attention? A comparison of two methodologies. Emotion, 6, 511516.

Järvinen-Pasley, A., Bellugi, U., Reilly, J., Mills, D. L., Galaburda, A., Reiss, A. L., et al. (2008). Defining the social phenotype in Williams syndrome: A model for linking gene, the brain, and behavior. Development and Psychopathology, 20, 1-35.

Jawaid, A., Schmolck, H., \& Schulz, P. E. (2008). Hypersociability in Williams syndrome: A role for the amygdala? Cognitive Neuropsychiatry, 13, 338-342.

Jones, W., Bellugi, U., Lai, Z., Chiles, M., Reilly, J., Lincoln, A., et al. (2000). Ii. Hypersociability in Williams syndrome. Journal of Cognitive Neuroscience, 12, 30-46.

Koster, E. H., Crombez, G., Verschuere, B., \& De Houwer, J. (2004). Selective attention to threat in the dot probe paradigm: Differentiating vigilance and difficulty to disengage. Behaviour Research and Therapy, 42, 1183-1192.

Krajcsi, A., Lukacs, A., Igacs, J., Racsmany, M., \& Pleh, C. (2009). Numerical abilities in Williams syndrome: Dissociating the analogue magnitude system and verbal retrieval. Journal of Clinical and Experimental Neuropsychology, 31, 439-446.

Leyfer, O. T., Woodruff-Borden, J., Klein-Tasman, B. P., Fricke, J. S., \& Mervis, C. B. (2006). Prevalence of psychiatric disorders in 4 to 16-year-olds with Williams syndrome. American Journal of Medical Genetics, Part B: (Neuropsychiatric Genetics), 141B, 615-622.

Lundqvist, D., Flykt, A., \& Ohman, A. (1998). The Karolinska Directed Emotional Faces (KDEF) CDROM. Karolinska: Department of Clinical Neuroscience, Psychology Section.

MacLeod, C., Rutherford, E., Campbell, L., Ebsworthy, G., \& Holker, L. (2002). Selective attention and emotional vulneravility: Assessing the causal bias of their association through the experimental maniulation of attentional bias. Journal of Abnormal Psychology, 111, 107-123.

Martens, M. A., Wilson, S. J., Dudgeon, P., \& Reutens, D. C. (2009). Approachability and the amygdala: Insights from Williams syndrome. Neuropsychologia, 47, 2446-2453. 
Mervis, C. B., Morris, C. A., Klein-Tasman, B. P., Bertrand, J., Kwitny, S., Appelbaum, L. G., et al. (2003). Attentional characteristics of infants and toddlers with Williams syndrome during triadic interactions. Developmental Neuropsychology, 23, 243-268.

Meyer-Lindenberg, A., Hariri, A. R., Munoz, K. E., Mervis, C. B., Mattay, V. S., Morris, C. A., et al. (2005). Neural correlates of genetically abnormal social cognition in Williams syndrome. Nature Neuroscience, 8, 991-993.

Mogg, K., Bradley, B. P., Miles, F., \& Dixon, R. (2004). Time course of attentional bias for threat scenes: Testing the vigilance-avoidance hypothesis. Cognition \& Emotion, 18, 689-700.

Mogg, K., Garner, M., \& Bradley, B. P. (2007). Anxiety and orienting of gaze to angry and fearful faces. Biological Psychology, 76, 163-169.

Mogg, K., Millar, N., \& Bradley, B. P. (2000). Biases in eye movements to threatening facial expressions in Generalized Anxiety Disorder and Depressive Disorder. Journal of Abnormal Psychology, 109, 695-704.

Mogg, K., Philippot, P., \& Bradley, B. P. (2004). Selective attention to angry faces in clinical Social Phobia. Journal of Abnormal Psychology, 113, 160-165.

Osborne, L.R., Li, Pober, M., Chitayat, D., Bodurtha, J., Mandel, A., et al. (2001). A 1.5 million-base pair inversion polymorphism in families with Williams-Beuren syndrome. Nature Genetics, $29,321-325$.

Peltola, M. J., Leppanen, J. M., Vogel-Farley, V. K., Hietanen, J. K., \& Nelson, C. J. (2009). Fearful faces but not fearful eyes alone delay attention disengagement in 7-month-old infants. Emotion, $9,560-565$.

Plesa-Skwerer, D., Borum, L., Verbalis, A., Crawford, N., Tager-Flusberg, H., Ciciolla, L., et al. (2009). Autonomic responses to dynamic displays of facial expressions in adolescents and adults with Williams syndrome. Social Cognitive and Affective Neuroscience, 4, 93-100. 
Plesa-Skwerer, D., Faja, S., Schofield, C., Verbalis, A., \& Tager-Flusberg, H. (2006). Perceiving facial and vocal expressions of emotion in individuals with Williams syndrome. American Journal on Mental Retardation, $111,15-26$.

Porter, M. A., Coltheart, M., \& Langdon, R. (2007). The neuropsychological basis of hypersociability in Williams and Down syndrome. Neuropsychologia, 45, 2839-2849.

Riby, D., \& Hancock, P. (2009a). Looking at movies and cartoons: Eye-tracking evidence from Williams syndrome and autism. Journal of Intellectual Disability Research, 53, 169-181.

Riby, D. M., \& Hancock, P. J. B. (2008). Viewing it differently: Social scene perception in Williams syndrome and autism. Neuropsychologia, 46, 2855-2860.

Riby, D. M., \& Hancock, P. J. B. (2009b). Do faces capture the attention of individuals with Williams syndrome or autism? Evidence from tracking eye movements. Journal of Autism \& Developmental Disorders, 39, 421-431.

Roy, A. K., Vasa, R. A., Bruck, M., Mogg, K., Bradley, B. P., Sweeney, M., et al. (2008). Attention bias toward threat in pediatric anxiety disorders. Journal of the American Academy of Child \& Adolescent Psychiatry, 47, 1189-1196.

Salemink, E., van den Hout, M. A., \& Kindt, M. (2007). Selective attention and threat: Quick orienting versus slow disengagement and two versions of the dot probe task. Behaviour Research and Therapy, 45, 607-615.

Sarimski, K. (1997). Behavioural phenotypes and family stress in three mental retardation syndromes. European Child \& Adolescent Psychiatry, 6, 26-31.

See, J., MacLeod, C., \& Bridle, R. (2009). The reduction of anxiety vulnerability through the modification of attentional bias: A real-world study using a home-based cognitive bias modification procedure. Journal of Abnormal Psychology, 118, 65-75.

Vicari, S., Bellucci, S., \& Carlesimo, G. A. (2006). Evidence from two genetic syndromes for the independence of spatial and visual working memory. Developmental Medicine and Child Neurology, 48, 126-131. 
Waters, A. M., Lipp, O. V., \& Spence, S. H. (2004). Attentional bias toward fear-related stimuli: An investigation with nonselected children and adults and children with anxiety disorders. Journal of Experimental Child Psychology, 89, 320-337.

Waters, A. M., Mogg, K., Bradley, B. P., \& Pine, D. S. (2008). Attentional bias for emotional faces in children with Generalized Anxiety Disorder. Journal of the American Academy of Child \& Adolescent Psychiatry, 47, 435-442.

Woodcock, R. W., \& Johnson, M. B. (Eds.). (1989, 1990). Woodcock-Johnson psycho-educational battery revised. Itascam IL: Riverside.

Yiend, J., \& Mathews, A. (2001). Anxiety and attention to threatening pictures. The Quarterly Journal of Experimental Psychology A: Human Experimental Psychology, 54A, 665-681. 
Table 1

Mean and standard deviation of age (years;months) and gender data for all groups

\begin{tabular}{|c|c|c|c|c|}
\hline & $\mathrm{N}$ & $\begin{array}{l}\text { Gender } \\
(\mathrm{M} ; \mathrm{F})\end{array}$ & $\begin{array}{c}\text { Chronological } \\
\text { Age }\end{array}$ & $\begin{array}{l}\text { Mental } \\
\text { Age }\end{array}$ \\
\hline & & & $M(s d)$ & $M(s d)$ \\
\hline Williams syndrome group & 16 & $9 ; 7$ & $19 ; 9$ & $8 ; 1$ \\
\hline & & & $(5 ; 3)$ & $(1 ; 1)$ \\
\hline Chronological age matched control group & 16 & $9 ; 7$ & $19 ; 9(5 . ; 5)$ & \\
\hline Mental age matched control group & 16 & $9 ; 7$ & $8 ; 2$ & \\
\hline
\end{tabular}

$(1 ; 3)$ 
Table 2

Mean and standard deviation of reaction time (ms) for each group and condition on the dot-probe task

\begin{tabular}{lccc}
\hline \multicolumn{1}{c}{ Condition } & Williams syndrome & Chronological Age & Mental Age \\
& $M(s d)$ & $M(s d)$ & $M(s d)$ \\
\hline Neutral-Neutral & $615.59(82.30)$ & $443.29(128.67)$ & $562.94(106.99)$ \\
Angry-congruent & $625.61(90.21)$ & $420.88(70.71)$ & $579.46(121.76)$ \\
Angry-incongruent & $623.55(79.89)$ & $415.01(66.43)$ & $577.59(124.90)$ \\
Happy-congruent & $613.34(86.71)$ & $419.27(72.44)$ & $582.87(121.03)$ \\
Happy-incongruent & $635.03(87.75)$ & $413.60(69.03)$ & $572.92(122.29)$ \\
Angry Bias & $-2.06(29.06)$ & $-5.87(12.05)$ & $-1.87(23.22)$ \\
Happy Bias & $21.69(43.36)$ & $-5.67(14.19)$ & $-9.95(19.50)$
\end{tabular}

Figure captions

Figure 1: Mean percentage correct on emotion recognition task for Williams syndrome group, chronological age matched control group and mental age matched control group. 


\section{Footnotes}

${ }^{1}$ The mean values shown in Table 2 suggest that the groups differed in their reaction time to neutral trials. As we were specifically interested in congruency by group interactions, rather than overall group effects, this difference in neutral RT should not affect the statistical analyses that follow. 